\title{
Super edge-magic models
}

\author{
S.C. López * \\ Departament de Matemàtica Aplicada IV \\ Universitat Politècnica de Catalunya, \\ Jordi Girona Salgado 1 \\ 08034 Barcelona, Spain \\ F. A. Muntaner-Batle \\ Graph Theory and Applications Research Group \\ School of Electrical Engineering and Computer Science \\ Faculty of Engineering and Built Environment \\ The University of Newcastle \\ NSW 2308 Australia \\ M. Rius-Font ${ }^{\dagger}$ \\ Departament de Matemàtica Aplicada IV \\ Universitat Politècnica de Catalunya, \\ Jordi Girona Salgado 1 \\ 08034 Barcelona, Spain
}

\begin{abstract}
In this paper, we generalize the concept of super edge-magic graph by introducing the new concept of super edge-magic models.
\end{abstract}

Keywords: Proper edge coloring, super edge-magic graph, super edge-magic model.

\section{Introduction}

By a graph we mean a simple graph, that is to say, a graph without loops or multiple edges. In general, for the terminology and notation found in this paper we follow either

\footnotetext{
*Supported by the Spanish Research Council under project MTM2008-06620-C03-01.

${ }^{\dagger}$ Supported by the Spanish Research Council under project MTM2008-06620-C03-01.
} 
$[2]$ or $[5]$.

In 1998 Enomoto, Lladó, Nakamigawa and Ringel defined super edge-magic labelings of graphs [3] inspired by the concept of edge-magic graphs introduced by Kotzig and Rosa in [6].

Let $G=(V, E)$ be a graph of order $p$ and size $q$. A bijective function $f: V \cup E \longrightarrow$ $\{i\}_{i=1}^{p+q}$ is an edge-magic labeling of $G$ if the sum $f(x)+f(x y)+f(y)=k$ for all $x y \in E$. In this case $G$ is called an edge-magic graph and $k$ is called either the valence or the magic sum of $f$. If $f$ has the extra property that $f(V)=\{i\}_{i=1}^{p}$, then $f$ is called a super edge-magic graph.

It is worthwhile mentioning that Achyra and Hedge introduced in 1991 the concept of strongly indexable graph, see [1], that turns out to be equivalent to the concept of super edge-magic graph, although the motivation for the definition is different, since while Enomoto et al. thought about constant sums, Achyra and Hegde thought about arithmetic progressions.

The next Lemma found in [4] shows how the concept of super edge-magic labeling can be thought in terms of arithmetic progressions and will be useful for the rest of the paper.

Lemma 1.1 A graph $G=(V, E)$ of order $p$ and size $q$ is super edge-magic if and only if there is a bijective function $g: V \longrightarrow\{i\}_{i=1}^{p}$ such that the set $S=\{g(x)+g(y)$ : $x y \in E\}$ is a set of exactly $q$ consecutive integers.

Generalizations of super edge-magic graphs (other than edge-magic graphs) can be found in the literature. For instance see [7]. In this paper we propose a different direction to generalize super edge-magic graphs. We do this by defining the concept of super edge-magic model. Before introducing this concept we need the following definition. Let $G$ be a graph of order $p$ and size $q$. A proper edge coloring of $G$ with $n$ different colors consists to assign $n$ colors to the edges of $G$, so that if two edges have a common vertex then they receive different colors. If $G$ is a graph with a proper edge coloring then $H$ is a rainbow subgraph of $G$ if it is found in $G$ so that each edge of $H$ receives a different color.

\section{Super edge-magic models}

Consider $n$ points on the plane, no three of them colineal, numbered with the numbers from 1 up to $n$. Join these $n$ points with $\left(\begin{array}{l}n \\ 2\end{array}\right)$ line segments, obtaining the complete graph $K_{n}$. Consider the function $g: E\left(K_{n}\right) \longrightarrow\{3,4, \ldots, 2 n-1\}$ that assigns to each edge of $K_{n}$ the sum of the values of two points incident with the edge. Then if we color these edges with $2 n-3$ different colors, so that two edges receive the same color if and 
only if they have the same image under $g$, we obtain a proper coloring of the edges of $K_{n}$. Finally we order these colors with a $2 n-3$ ordered tuple, according with the numerical value associated with that color. That is to say, the color associated with 3 is the first color of the tuple, the color associated with 4 is the second color of the tuple and so on until the color associated with $2 n-1$ which is the last color of the tuple. Then the following observation is trivial:

Observation 2.1 A graph of order $n$ is super edge-magic if and only if it can be found as a rainbow subgraph of $K_{n}$ colored as described above, when the colors of the edges are consecutive in the tuple.

Let us see the next example that appears in Figure 1.

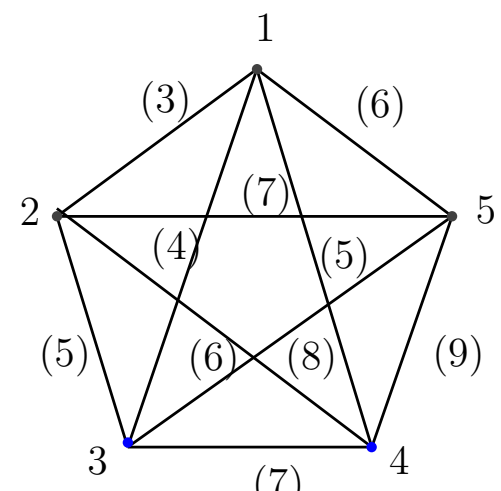

$(7)$

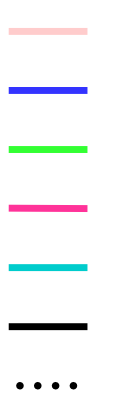

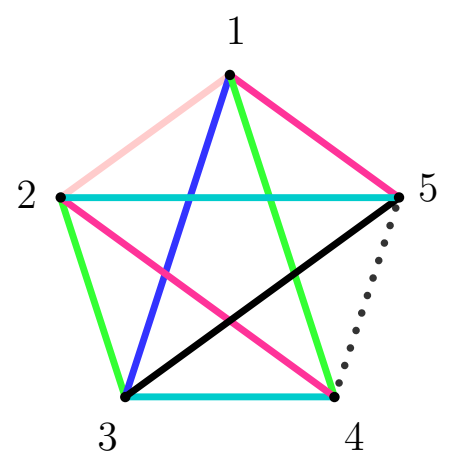

Figure 1: A proper edge-coloring of $K_{5}$ with an order in the colors.

Then, for instance, $C_{5}$ is super edge-magic, see Figure 2.

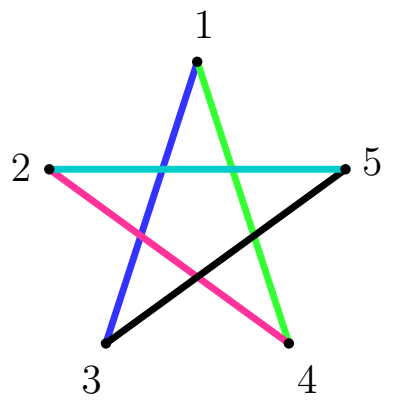

Figure 2: $C_{5}$ as a rainbow subgraph of $K_{5}$ with consecutive colors. 
The goal of this section is to generalize this idea to the concept of super edge-magic model.

Let $m, n \in \mathbb{N}$. A super edge-magic $m$-model of order $n$ is a proper edge coloring of the edges of $K_{n}$ with $m$ colors, together with a $m$-ordered tuple of the $m$ colors used in the coloring. A graph $G$ of order $n$ is super edge-magic with respect to a super edge-magic $m$-model if and only if $G$ can be found as a rainbow subgraph of $K_{n}$ and the colors of the edges of $G$ are consecutive in the $m$-tuple of the model.

Example 2.2 Let us see an example of an m-model. The next Figure shows a super edge-magic 5-model of order 6 with the order in the left side, and a super edge-magic triangle with respect to it on the right side.
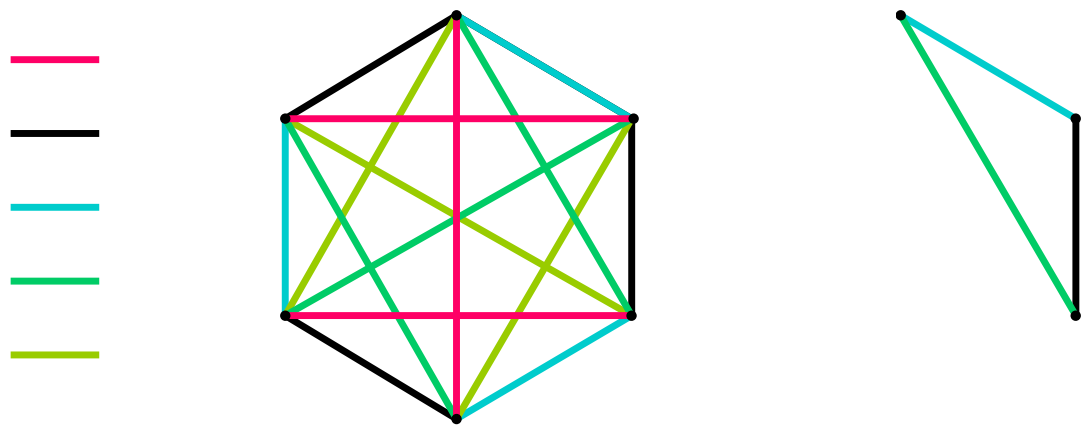

Figure 3: A super edge-magic 5-model and a super edge-magic $C_{3}$ with respect to it.

The following are two easy observations:

Observation 2.3 If a graph $G=(V, E)$ is super edge-magic with respect to an $m$ model then the size of the graph is at most the number of different colors. That is, $|E| \leq m$.

Observation 2.4 Every rainbow subgraph of size $m$ of a super edge-magic $m$-model is super edge-magic with respect to the model.

\section{Results on super edge-magic m-models}

Let $m$ be an even number. In this section we answer the following question: can we find a super edge-magic $m$-model of order $m$ such that no 2-regular graph of order $m$ is super edge-magic with respect to this model?. 
We answer this question in an affirmative way. We begin by describing the model, and once we have the description, we will prove that it does not contain any 2-regular rainbow spanning subgraph.

\section{Description of the model}

Let $n$ be an even number. Place $n$ points forming the vertices of a regular $n$-gon in the plane, and join these vertices with $\left(\begin{array}{l}n \\ 2\end{array}\right)$ straight line segments forming the complete graph $K_{n}$. The coloring of the edges is given in the following way:

Two edges receive the same color if and only if they belong to parallel lines.

In light of the last observation of the previous section, we do not need to specify any ordered tuple for the colors, since it is clear that this coloring uses exactly $n$ colors. It is also clear that the coloring is a proper coloring.

From now on we will denote this coloring of order $n$ by $\mu_{n}$.

Example 3.1 The proper coloring of $\mu_{4}$ and $\mu_{6}$ are showed in Figure 4.
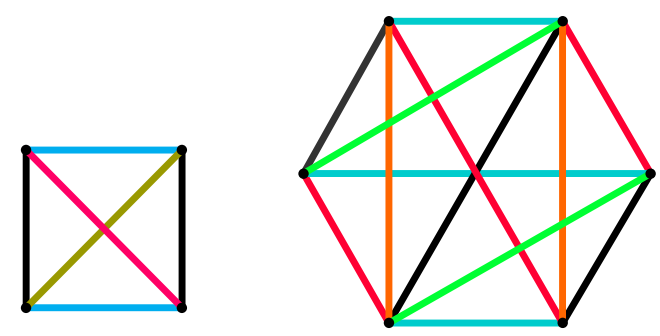

Figure 4: The 4-model $\mu_{4}$ and the 6-model $\mu_{6}$,

Next, we prove the following result:

Theorem 3.2 Let $n$ be an even number. No 2-regular graph of order $n$ is super edgemagic with respect to $\mu_{n}$.

Proof.

Consider the model $\mu_{n}$. Label the vertices of $\mu_{n}$ with the numbers from 1 up to $n$ in increasing order in the counterclockwise sense. Then consider the function $g$ : $E\left(K_{n}\right) \longrightarrow \mathbb{N}$ that assigns to each edge the sum of the labels of its terminal vertices, and assume that $H$ is any 2-regular spanning subgraph of $\mu_{n}$. Then :

$$
\sum_{e \in E(H)} g(e)=2 \sum_{i=1}^{n} i=n(n+1)
$$


since each vertex belongs to exactly two edges of $H$.

Next, we will describe the possible values that the labeling $g$ assigns to edges of the same colors. We can see this in the next table, where we denote the colors by $c_{1}, c_{2}, \ldots, c_{n}$.

\begin{tabular}{|c|cc|}
\hline Colors & Possible values for edges of color $c_{i}$ assigned by $g$ \\
\hline$c_{1}$ & 3, & $3+n$ \\
$c_{2}$ & 4, & $4+n$ \\
$c_{3}$ & 5, & $5+n$ \\
$\vdots$ & $\vdots$ & $\vdots$ \\
$c_{n-3}$ & $n-1$, & $2 n-1$ \\
$c_{n-2}$ & $n$ \\
$c_{n-1}$ & $n+1$ \\
$c_{n}$ & \multicolumn{2}{|c}{$n+2$} \\
\hline
\end{tabular}

Assume to the contrary that $H$ is a rainbow 2-regular spanning subgraph of $\mu_{n}$. Then the sum of the values assigned by $g$ to the edges of $H$ is obtained adding the numbers $n, n+1, n+2$ and exactly one number in each of the following sets: $\{3,3+n\},\{4,4+$ $n\}, \ldots,\{n-1,2 n-1\}$. Assume that this sum contains exactly $k$ numbers in the set $\{n+3, n+4, \ldots, 2 n-1\}$. Then we have the following equality:

$$
\frac{n(n+5)}{2}+k n=n(n+1) \Longrightarrow 2 k=2(n+1)-(n-1)-6
$$

Hence we have that an odd number is equal to an even number, and therefore the desired contradiction has been reached.

From the previous discussion it is easy to obtain the following.

Let $n$ be an even number. Consider $n$ points in the plane that are placed forming the vertices of a regular $n$-gon. Assume that with this configuration, we create not necessarily simple polygons, by joining all $n$ vertices with straight segments, so that each vertex belongs to exactly one of these polygons formed. Call a configuration of this type $C^{(n)}$. Then we have.

Corollary 3.1 For $n$ even, any $C^{(n)}$ contains at least two sides that belong to parallel lines.

\section{Conclusions and open problems}

In this paper we have generalized the concept of super edge-magic graphs to the concept of super edge-magic graphs with respect to a model. For $n$ even, we have found an $n$ 
model of order $n$, namely $\mu_{n}$, so that no 2-regular graph of order $n$ is super edge magic with respect to $\mu_{n}$.

Many different lines of research may be followed from the concept of super edge-magic graphs with respect to a model, and next we propose some questions that we feel may be interesting to consider for further research.

Question 4.1 For $n$ even, find an $n$-model of order $n$, namely $\nu_{n}$, so that at least one 2 -regular graph of order $n$ is super edge-magic with respect to $\nu_{n}$, or prove that such model $\nu_{n}$ does not exist.

Question 4.2 For $n$ even, find an $n$-model of order $n$ so that each 2-regular graph of order $n$ is super edge-magic with respect to the model.

Question 4.3 For $n \in \mathbb{N}$, find an $n$-model of order $n$ that maximizes the number of spanning 2-regular rainbow subgraphs that the model contains. Find this maximum.

Question 4.4 Let $G$ be a graph of order $p$ and size $q$. Find, when possible, a q-model of order $p$ that does not contain any rainbow subgraphs isomorphic to $G$.

Question 4.5 Let $G$ be a graph of order $p$ and size $q$. Find a q-model of order $p$ that maximizes the number of rainbow subgraphs isomorphic to $G$.

Next we make a remark about Question 1 and Question 2. For $p=4$, the only 2-regular graph of order 4 is the cycle $C_{4}$. Next we show all possible proper edge colorings of the edges of $K_{4}$ with 4 colors, and it is easy to check that none of these colorings contain a rainbow $C_{4}$.
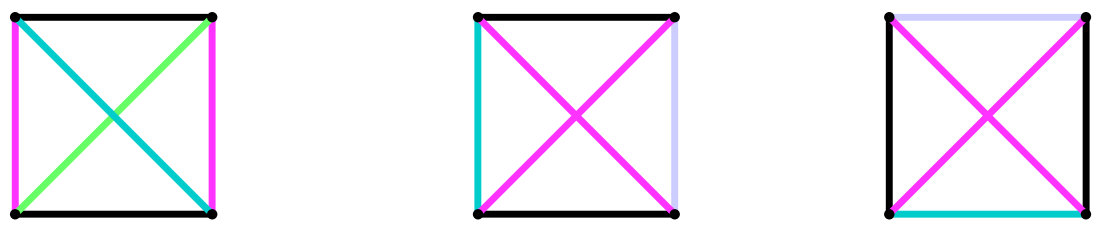

Figure 5: All possible proper edge colorings of the edges of $K_{4}$ with 4 colors

However for general even $n$ this is not true. For instance the proper coloring of the edges of $K_{6}$ with 6 colors that appears in Figure 6 contains a $C_{6}$ as a rainbow subgraph. 


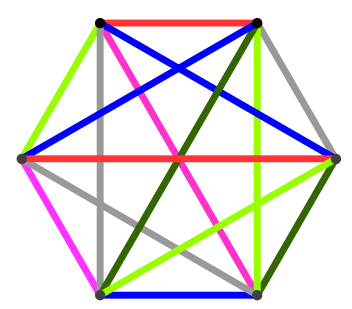

Figure 6: A proper edge coloring of $K_{6}$.

Furthermore, by exploring all cases, we have checked that, up to isomorphisms, this is the only proper coloring of the edges of $K_{6}$ with six colors that contains a rainbow subgraph $C_{6}$ and no such coloring contains $2 C_{3}$ as a rainbow subgraph.

In order to conclude this section, we observe that the idea of relating labelings with rainbow subgraphs can be extended to other types of labelings. For instance when we think about graceful labelings of trees (see [5] for further information on graceful labelings and the graceful tree conjecture), then we notice that they can be thought of rainbow spanning subtrees of the following coloring (not necessarily proper) of the complete graph $K_{n}$.

Label the vertices of $K_{n}$ with the numbers $0,1,2, \ldots, n-1$ and assign to each edge of $K_{n}$ the value $|i-j|$, where $i, j$ are the labels of the vertices incident with each edge. Color each edge of $K_{n}$ with a color corresponding to the assigned value. It is clear that a tree of order $n$ is graceful if and only if it can be found as a rainbow subgraph of this colored $K_{n}$. Therefore, we also propose to find and explore other relations of this type for further research.

\section{References}

[1] B.D. Acharya and S.M. Hegde, Strongly indexable graphs, Discrete Math. 93 (1991), 123-129.

[2] G. Chartrand and L. Lesniak, Graphs and Digraphs, second edition. Wadsworth \& Brooks/Cole Advanced Books and Software, Monterey (1986).

[3] H. Enomoto, A. Lladó, T. Nakamigawa and G. Ringel, Super edge-magic graphs, SUT J. Math. 34 (1998), 105-109.

[4] R.M. Figueroa-Centeno, R. Ichishima and F.A. Muntaner-Batle, The place of super edge-magic labelings among other classes of labelings, Discrete Math. 231(1-3) (2001), 153-168. 
[5] J.A. Gallian, A dynamic survey of graph labeling, Electron. J. Combin. 16 (2009), $\sharp$ DS6.

[6] A. Kotzig and A. Rosa, Magic valuations of finite graphs, Canad. Math. Bull. 13 (1970), 451-461.

[7] Lladó, A. and Gutiérrez, Magic coverings, J. Comb. Math. and Comb 55 (2005), 43-56. 